\title{
Vector Variational Inequalities In $G$-Convex Spaces
}

\author{
Maryam Salehnejad and Mahdi Azhini
}

\begin{abstract}
In this paper, we study some existence theorems of solutions for vector variational inequality by using the generalized KKM theorem. Also, we investigate the properties of solution set of the Minty vector variational inequality in $G$-convex spaces. Finally, we prove the equivalence between a Browder fixed point theorem type and the vector variational inequality in G-convex spaces.
\end{abstract}

\section{Introduction}

The variational inequality theory, which was introduced by Stampacchia [19], provides very useful techniques for studying problems in optimization, transportation, economics, contact problems in elasticity and other branches of mathematics. The existence of solutions and properties of solutions set for variational inequality were studied by many authors in linear topological spaces (see $[14,15])$. These results are dependent on the linear structure of spaces. Since 1987, some authors have introduced a new concept of convexity on spaces without any linear structure. So, these problems generalized to nonlinear spaces (see [17, 21, 22]). Xian Wu et al [24] presented the character of solution sets for nonlinear variational inequalities in $H$-spaces in 1999. Additionally, J.Wang [23] proved the existence of solutions of a type of variational inequality for scalar functions in the framework of $F C$-spaces in 2012. Another type of generalizations of variational inequalities is generalization to vector case. The theory of vector variational inequalities was initiated by Giannessi [5] in finite dimensional Euclidean spaces. In recent years, vector variational inequalities have extended because of their broad applications such as vector optimization, vector equilibria, vector traffic equilibria and other problems of practical interest (see $[1,4,6])$. For instance, Tran Thi Mai and Do Van Luu [11] in 2018, studied Fritz John necessary conditions for weakly efficient solutions of nonsmooth vector variational inequality problems with constraints 2010 Mathematics Subject Classification. 49J40, 49J20, 90C33.

Key words and phrases. vector variational inequality; Minty vector variational inequality; generalized KKM mapping; $G$-convex spaces; monotone functions..

Corresponding author: Mahdi Azhini. 
in terms of convexificators. Moreover, T. Chen et al [3] in 2019, investigated new existence theorems for vector equilibrium by vector variational inequality theorems. Let $E$ be a nonempty subset of a Hausdorff topological G-convex space and $f: E \rightarrow V$ and $\phi: E \times E \rightarrow V$ are vector valued functions. In this article, we generalize the results presented by X.Wu et al [24] and J.Wang [23] to vector valued functions on $G$-convex spaces. Particularly, we study the existence of solutions for the following vector variational inequality (in short VVI) by using the generalized KKM Theorem.

$$
\phi(x, y)+f(y)-f(x) \in P
$$

Additionally, we investigate the Minty type theorem and the properties of the solutions set for vector variational inequality (1.1) in G-convex spaces. This paper is organized as follows. In section 2, we present some preliminaries and definitions which are required. In the third section, we prove the existence theorem for vector variational inequality problem in G-convex spaces and also examine the characteristics of the solutions. Finally, in section 4 , we prove the equivalence between the Browder fixed point Theorem and the vector variational problem (1.1) in G-convex spaces as an application.

\section{Preliminaries}

Let $X$ be a topological space and $E$ be a nonempty subset of $X$. We denote by $\langle E\rangle$, the family of all nonempty finite subsets of $E$. Let $\Delta_{n}$ be the standard $n$-simplex $\left(e_{1}, \ldots, e_{n}\right)$ in $R^{n+1}$. If $J$ is a nonempty subset of $\{0,1, \ldots, n\}$, we denote by $\Delta_{J}$ the convex hull of the vertices $\left\{e_{j}, j \in J\right\}$. The following notion of a generalized convex (or $G$-convex) space was introduced by Park and Kim [12]. Let $X$ be a topological space and $D$ is a nonempty set, $(X, D ; \Gamma)$ is said to be a $G$ convex space if for each $A=\left\{a_{0}, \ldots, a_{n}\right\} \in\langle D\rangle$, there exists a subset $\Gamma(A)=\Gamma_{A}$ of $X$ and a continuous function $\phi_{A}: \Delta_{n} \rightarrow \Gamma(A)$ such that $J \subset A$ implies $\phi_{A}\left(\Delta_{J}\right) \subset \Gamma(J)$.

When $D \subset X,(X, D ; \Gamma)$ will be denoted by $(X \supset D ; \Gamma)$ and if $X=D$, we write $(X ; \Gamma)$ in place of $(E, E ; \Gamma)$. For a $G$-convex space $(X \supset D ; \Gamma)$,

1. a subset $Y$ of $X$ is said to be $\Gamma$-convex if for each $N \in\langle D\rangle, N \subset Y$ implies $\Gamma_{N} \subset Y$;

2. the $\Gamma$-convex hull of a subset $Y$ of $X$, denoted by $\Gamma-\operatorname{Co}(Y)$, is defined by

$$
\Gamma-\operatorname{Co}(Y)=\bigcap\{Z \subset X: Z \text { is a } \Gamma \text {-convex subset containing } Y\} .
$$

There are a lot of examples of $G$-convex spaces:

Example 1. Every vector space $X$ with convex hull is a $G$-convex space. Especially $(\mathbb{R}, C o)$ is a $G$-convex space. 
Example 2. If $X$ is a convex subset of a vector space, $D \subset X$, and $X$ has a topology such that each $\Gamma_{A}$ is the convex hull of $A \in\langle D\rangle$ equipped with the Euclidean topology, then $(X, D ; \Gamma)$ becomes a convex space generalizing the one due to Lassonde [10]. $(X, D ; \Gamma)$ is a $G$-convex space.

Example 3. If $X=D$ and $\Gamma_{A}$ is assumed to be contractible or, more generally, infinitely connected (that is, $\mathrm{n}$-connected for all $n \geq 0$ ) and if for each $A, B \in\langle X\rangle, A \subset B$ implies $\Gamma_{A} \subset \Gamma_{B}$, then $(X ; \Gamma)$ becomes an $\mathrm{H}$-space due to Horvath $[7,8]$. $\mathrm{H}$-spaces are examples of $G$-convex spaces.

Remark 1. It is easily to see:

1. For any $Y \subseteq X, Y \subseteq \Gamma-\operatorname{Co}(Y)$.

2. $Y \subseteq X$ is $\Gamma$-convex $\Leftrightarrow Y=\Gamma-\operatorname{Co}(Y)$.

3. For any $Y \subseteq X, \Gamma-\operatorname{Co}(Y)$ is $\Gamma$-convex.

4. If $Y \subseteq Z \subseteq X, \Gamma-\operatorname{Co}(Y) \subseteq \Gamma-\operatorname{Co}(Z)$.

5. For any $Y \subseteq X$ finite, $\Gamma_{Y} \subseteq \Gamma-\operatorname{Co}(Y)$.

For further details, see $[12,13]$.

Definition 1 ([20]). Let $Y$ be a nonempty set and $E$ be a nonempty subset of a $G$-convex space $(X, D ; \Gamma) . T: Y \rightarrow 2^{E}$ is called a generalized KKM mapping if for any finite set $\left\{y_{0}, y_{1}, \ldots, y_{n}\right\} \subset$ $Y$, there exists $\left\{x_{0}, x_{1}, \ldots, x_{n}\right\} \in\langle E \cap D\rangle$ such that for any subset $\left\{x_{i_{0}}, x_{i_{1}}, \ldots, x_{i_{k}}\right\} \subset$ $\left\{x_{0}, \ldots, x_{n}\right\}, 0 \leq k \leq n$, we have

$$
\Gamma\left(\left\{x_{i_{0}}, \ldots, x_{i_{k}}\right\}\right) \subset \bigcup_{j=0}^{k} T\left(y_{i_{j}}\right)
$$

Let $V$ be a real vector space, a nonempty subset $P$ of $V$ is called a convex cone if $\lambda P \subset P$ for all $\lambda \geq 0$ and $P+P \subset P$. The cone $P$ is called pointed if $P \cap(-P)=\{0\}$. Also $P$ is solid if int $P \neq \emptyset$ (where int $P$ denotes the interior of $P$ ). The partial order $\leq_{P}$ on $V$ induced by a pointed convex cone $P$ is defined by $x \leq_{P} y$ if and only if $y-x \in P$ for $x, y$ in $V$. The notation $x<_{P} y$ means $x \leq_{P} y$ and $x \neq y$.

The weak order $<_{P}$ on ordered vector space $(V, P)$ with int $P$ is defined as $x<y$ if and only if $y-x \in$ int $P$ for $x, y$ in $V$.

Definition 2. Let $(X ; \Gamma)$ be a $G$-convex space and $E$ be a nonempty and $\Gamma$-convex subset of $X$ and $(V, P)$ be an ordered vector space. Let $\gamma \in V$ and $\phi: E \times E \rightarrow V$. Then 
1. $\phi$ is said to be cone- $\gamma-q u a s i-c o n v e x$ (concave) related to the second variant if $\{y \in E$ : $\phi(x, y) \notin \gamma+P\}(\{y \in E: \phi(x, y) \in \gamma+P\})$ is a $\Gamma$-convex subset of $E$ for any $x \in E$. We say that $\phi$ is cone-quasi-convex (quasi-concave) related to the second variant if for each $\gamma \in V$ and for any $x \in E, \phi$ is cone- $\gamma$-quasi-convex (quasi-concave).

2. $\phi$ is said to be cone- $\gamma$-generalized quasi-convex (concave) in second variant, if for each $\left\{y_{0}, \ldots, y_{n}\right\} \subset E$, there exists a finite subset $\left\{x_{0}, x_{1}, \ldots, x_{n}\right\} \subset E$ such that for any subset $\left\{x_{i_{0}}, \ldots, x_{i_{k}}\right\} \subset\left\{x_{0}, \ldots, x_{n}\right\}$ and $x^{*} \in \Gamma-\operatorname{Co}\left\{x_{i_{0}}, \ldots, x_{i_{k}}\right\}$, there exists $j \in\{0, \ldots, k\}$ such that

$$
\begin{aligned}
& \phi\left(x^{*}, y_{i_{j}}\right) \in \gamma+P \\
& \left(\phi\left(x^{*}, y_{i_{j}}\right) \in \gamma-P\right)
\end{aligned}
$$

3. $f: E \rightarrow V$ is called strongly cone-quasi-convex if $f$ is cone-quasi-convex and for each $\gamma \in V$, if $f\left(y_{1}\right) \notin \gamma+P$ and $f\left(y_{2}\right) \notin \gamma+$ int $P$ implies that $f(y) \notin \gamma+P$ for all $y \in \Gamma-\operatorname{Co}\left\{y_{1}, y_{2}\right\} \backslash\left\{y_{1}, y_{2}\right\}$.

4. $f: E \rightarrow V$ is called cone-w-convex iff for each $\gamma \in V$, if $f\left(y_{1}\right) \notin \gamma+P$ and $f\left(y_{2}\right) \notin$ $\gamma+$ int $P$ implies that $f(y) \notin \gamma+P$ for all $y \in \Gamma-\operatorname{Co}\left\{y_{1}, y_{2}\right\} \backslash\left\{y_{1}, y_{2}\right\}$.

Theorem 2.1 (KKM Lemma, [9]). Let $D$ be the set of vertices of $n-\operatorname{simplex} \Delta_{N}$ and $F: D \multimap \Delta_{N}$ a KKM map with closed (respectively open) values. Then $\bigcap_{x \in D} F(x) \neq \emptyset$.

Definition 3. Let $X$ be a topological space. We say that $Y \subseteq X$ is compactly closed subset of $X$, if for every compact set $K \subset X$, the set $K \cap Y$ is closed in $K$.

Theorem 2.2. Let $E$ be a nonempty $\Gamma$-convex subset of a $G$-convex space $(X ; \Gamma)$ and $G: E \rightarrow 2^{X}$ be such that for any $y \in E, G(y)$ is compactly closed. Then:

1. If $G$ is a generalized KKM mapping, then the family of sets $\{G(y): y \in E\}$ has the finite intersection property.

2. If the family $\{G(y): y \in E\}$ has the finite intersection property and $\Gamma(x)=\{x\}$ for each $x \in X$, then $G$ is a generalized KKM mapping.

Proof. (1) Let $G: E \rightarrow 2^{X}$ is a generalized KKM mapping with compactly closed values, and suppose $\left\{y_{0}, \ldots, y_{n}\right\}$ be arbitrary finite subset of $E$. Since $G$ is a generalized KKM mapping, there exists finite subset $\left\{x_{0}, \ldots, x_{n}\right\} \subset X$ such that for any subset $\left\{x_{i_{0}}, \ldots, x_{i_{k}}\right\} \subset\left\{x_{0}, \ldots, x_{n}\right\}$,

$$
\Gamma\left(\left\{x_{i_{0}}, \ldots, x_{i_{k}}\right\}\right) \subset \bigcup_{j=0}^{k} G\left(y_{i_{j}}\right),
$$


especially, $\Gamma\left(\left\{x_{0}, \ldots, x_{n}\right\}\right) \subset \bigcup_{i=0}^{n} G\left(y_{i}\right)$. As $(X ; \Gamma)$ is $G$-Convex space, there exists a continuous $\operatorname{map} \phi_{n}: \Delta_{n} \rightarrow \Gamma\left(\left\{x_{0}, x_{1}, \ldots, x_{n}\right\}\right)$ such that

$$
\phi_{n}\left(\Delta_{J}\right) \subset \Gamma(J) \quad \forall J \subset\left\{x_{0}, \ldots, x_{n}\right\}
$$

especially, $\phi_{n}\left(\Delta_{n}\right) \subseteq \Gamma\left(\left\{x_{0}, x_{1}, \ldots, x_{n}\right\}\right) \subseteq \bigcup_{i=0}^{n} G\left(y_{i}\right)$. So,

$$
\Delta_{n} \subset \bigcup_{i=0}^{n} \phi_{n}^{-1}\left(G\left(y_{i}\right) \cap \phi_{n}\left(\Delta_{n}\right)\right) .
$$

Since $G\left(y_{i}\right) \cap \phi_{n}\left(\Delta_{n}\right)$ is closed in the compact subset $\phi_{n}\left(\Delta_{n}\right)$ of $\Gamma\left(\left\{x_{0}, \ldots, x_{n}\right\}\right)$, then $\phi_{n}^{-1}\left(G\left(y_{i}\right) \cap\right.$ $\left.\phi_{n}\left(\Delta_{n}\right)\right)$ is closed in $\Delta_{n}$. Note that $F: \Delta_{J} \rightarrow 2^{\Delta_{n}}$, which is defined by $e_{i} \multimap \phi_{n}^{-1}\left(G\left(y_{i}\right) \cap\right.$ $\left.\phi_{n}\left(\Delta_{n}\right)\right)$, by (2.2) is a KKM map.

Hence, by the KKM lemma, we have:

$$
\bigcap_{i=0}^{n} \phi_{n}^{-1}\left(G\left(y_{i}\right) \cap \phi_{n}\left(\Delta_{n}\right)\right) \neq \phi
$$

which easily shows $\bigcap_{i=0}^{n} G\left(y_{i}\right) \neq \phi$.

(2) Suppose that $\{G(y): y \in E\}$ has the finite intersection property, so for $y_{0}, \ldots, y_{n} \in E$, we have $\bigcap_{i=0}^{n} G\left(y_{i}\right) \neq \phi$. Consider $x^{\prime} \in \bigcap_{i=0}^{n} G\left(y_{i}\right)$ and let $x_{i}=x^{\prime}$ for $i=0,1, \ldots, n$. For any finite nonempty subset $\left\{x_{i_{0}}, \ldots, x_{i_{k}}\right\} \subset\left\{x_{0}, \ldots, x_{n}\right\}$, we conclude

$$
\Gamma\left(\left\{x_{i_{0}}, \ldots, x_{i_{k}}\right\}\right)=\left\{x^{\prime}\right\} \subset \bigcap_{i=0}^{n} G\left(y_{i}\right) \subset \bigcup_{j=0}^{k} G\left(y_{i_{j}}\right) .
$$

this means that $G: E \rightarrow 2^{X}$ is generalized KKM map.

We can easily prove the following result using Theorem 2.2 .

Theorem 2.3. Suppose $G: E \rightarrow 2^{X}$ is a generalized KKM mapping and compactly closed valued and for some $M \in\langle E\rangle, \bigcap_{x \in M} G(x)$ is compact, then $\bigcap_{x \in E} G(x) \neq \phi$.

\section{Main Results}

In sequel, suppose that $X$ is a Hausdorff topological space, $(X ; \Gamma)$ is a $G$-convex space and $E$ is a nonempty $\Gamma$-convex subset of $X$. Also, $(V, P)$ is an ordered topological vector space and $P$ is a closed pointed convex cone such that int $P \neq \phi$. 
In this section, we present some existence results for vector variational inequality (1.1) and then we will consider the Minty type theorem and monotone vector variational inequality.

Theorem 3.1. Suppose that $f: E \rightarrow V, \phi: E \times E \rightarrow V$ and $g(x, y)=\phi(x, y)+f(y)-f(x)$ are functions satisfying:

1. $g(x, y)$ is cone-0-generalized quasi-convex in $y$.

2. For every $y \in E,\{x \in E: g(x, y) \in P\}$ is compactly closed.

3. There exists a compact subset $K$ of $X$ and $y^{*} \in E \cap K$ such that

$$
g\left(x, y^{*}\right) \in-\text { int } P \quad \text { for all } x \in E \backslash K \text {. }
$$

Then VVI (1.1) has a solution in $E \cap K$, i.e., there exists an $\bar{x} \in E \cap K$ such that

$$
g(\bar{x}, y)=\phi(\bar{x}, y)+f(y)-f(\bar{x}) \in P \quad \text { for all } y \in E
$$

Proof. For each $y \in E$, let $G(y)=\{x \in E: g(x, y) \in P\}$. Then $G$ is a set-valued mapping from $E$ into $2^{E}$.

Condition (1) imply that $G$ is a generalized KKM mapping. Because for any finite set $\left\{y_{0}, \ldots, y_{n}\right\} \subset$ $E$, there exists $\left\{x_{0}, \ldots, x_{n}\right\} \subset E$ such that for any subset $\left\{x_{i_{0}}, \ldots, x_{i_{k}}\right\} \subset\left\{x_{0}, \ldots, x_{n}\right\}$ and any $x^{*} \in \Gamma-\operatorname{Co}\left\{x_{i_{0}}, \ldots, x_{i_{k}}\right\}$, there exists $j \in\{0, \ldots, k\}$ such that $g\left(x^{*}, y_{i_{j}}\right) \in P$. According to the definition of $G$, we have $x^{*} \in G\left(y_{i_{j}}\right)$.

As $x^{*} \in \Gamma-\operatorname{Co}\left\{x_{i_{0}}, \ldots, x_{i_{k}}\right\}$ is arbitrary, we have

$$
\Gamma-\operatorname{Co}\left\{x_{i_{0}}, \ldots, x_{i_{k}}\right\} \subset \bigcup_{j=0}^{k} G\left(y_{i_{j}}\right) .
$$

So, by Remark 1, $\Gamma\left\{x_{i_{0}}, \ldots, x_{i_{k}}\right\} \subset \Gamma-\operatorname{Co}\left\{x_{i_{0}}, \ldots, x_{i_{k}}\right\} \subset \bigcup_{j=0}^{k} G\left(y_{i_{j}}\right)$. This means that $G$ : $E \rightarrow 2^{E}$ is a generalized KKM mapping. By assumption (2), $G$ is a compactly closed valued map. From condition (3) and having $P$ is pointed, we have $x \notin G\left(y^{*}\right)$ for all $x \in E \backslash K$, so $G\left(y^{*}\right) \subset K$. Considering that $G$ is compactly closed valued, then $G\left(y^{*}\right)$ is compact. Hence, by Theorem 2.3, $\bigcap_{y \in E} G(y) \neq \phi$. Therefore, there exist an $\bar{x} \in \bigcap_{y \in E} G(y) \subset G\left(y^{*}\right) \subset K$ such that

$$
g(\bar{x}, y) \in P \quad \text { for all } y \in E
$$

i.e.

$$
\phi(\bar{x}, y)+f(y)-f(\bar{x}) \in P \quad \text { for all } y \in E,
$$

so the solutions of VVI (1.1) is in $E \cap K$. 
Remark 2. If the condition 3 in theorem 3.1 replaced by the following condition:

$(3)^{\prime}$ For some $y_{0} \in E,\left\{x \in E: \phi\left(x, y_{0}\right)+f\left(y_{0}\right)-f(x) \in P\right\}$ is compact.

Then VVI (1.1) has a solution in $E$. Because according to the proof of Theorem 3.1, $G$ is a generalized KKM mapping with compactly closed valued. With regard to condition $(3)^{\prime}$ and Theorem 2.3 , the result is obtained.

Definition 4 ([18]). A function $f: E \rightarrow V$ is said to be lower semi-continuous (upper semicontinuous) if for every $\gamma \in V,\{x \in E: f(x) \in \gamma-P\}(\{x \in E: f(x) \in \gamma+P\})$ is closed in E.

The following is a generalization of Theorem 3.2 of [23] to vector case and for $G$-convex spaces.

Theorem 3.2. Suppose $f: E \rightarrow V$ and $\phi: E \times E \rightarrow V$ with $\phi(x, x) \in P$ for all $x \in E$ and the following conditions satisfy:

1. There exist a compact subset $K$ of $X$ and $y^{*} \in E \cap K$ such that

$$
\phi\left(x, y^{*}\right)+f\left(y^{*}\right)-f(x) \in-\operatorname{int} P \quad \text { for all } x \in E \backslash K \text {. }
$$

2. $f(y)+\phi(x, y)$ is cone-quasi-convex related to $y$.

3. $f(x)-\phi(x, y)$ is lower semi-continuous related to $x$.

Then VVI (1.1) has a solution in $E \cap K$.

Proof. We first prove that the set-valued mapping $G: E \rightarrow 2^{E}$ defined by $G(y)=\{x \in$ $E: f(y)+\phi(x, y)-f(x) \in P\}$ is a generalized KKM mapping. Suppose that this is not, so, there exist $\left\{y_{0}, \ldots, y_{n}\right\} \subset E$ such that for any $\left\{x_{0}, \ldots, x_{n}\right\} \subset E$, there exist $\left\{x_{i_{0}}, \ldots, x_{i_{k}}\right\} \subset$ $\left\{x_{0}, \ldots, x_{n}\right\}$ such that

$$
\Gamma\left\{x_{i_{0}}, \ldots, x_{i_{k}}\right\} \not \subset \bigcup_{j=0}^{k} G\left(y_{i_{j}}\right) .
$$

If we assume $x_{i}=y_{i}, i=0, \ldots, n$, then there exist $\left\{y_{i_{0}}, \ldots, y_{i_{k}}\right\} \subset\left\{y_{1}, \ldots, y_{n}\right\}$ such that

$$
\Gamma\left\{y_{i_{0}}, \ldots, y_{i_{k}}\right\} \not \subset \bigcup_{j=0}^{k} G\left(y_{i_{j}}\right),
$$

therefore there exist $x^{*} \in \Gamma\left\{y_{i_{0}}, \ldots, y_{i_{k}}\right\}$ such that $x^{*} \notin G\left(y_{i_{j}}\right)$ for $j=0, \ldots, k$. Since $f(x)+$ $\phi(x, y)$ is cone-quasi-convex related to $y$ and $\left\{y_{i_{0}}, \ldots, y_{i_{k}}\right\} \subset\left\{y \in E ; f(y)+\phi\left(x^{*}, y\right) \notin\right.$ $\left.f\left(x^{*}\right)+P\right\}$, then $\Gamma\left\{y_{i_{0}}, \ldots, y_{i_{k}}\right\} \subset\left\{y \in E ; f(y)+\phi\left(x^{*}, y\right) \notin f\left(x^{*}\right)+P\right\}$. So $x^{*} \in\{y \in E$ : 
$\left.f(y)+\phi\left(x^{*}, y\right) \notin f\left(x^{*}\right)+P\right\}$. Hence $f\left(x^{*}\right)+\phi\left(x^{*}, x^{*}\right) \notin f\left(x^{*}\right)+P$ i.e. $\phi\left(x^{*}, x^{*}\right) \notin P$, this contradicts $\phi(x, x) \in P$ for all $x \in E$. Therefore, $G$ is a generalized KKM mapping.

According to assumption (1), $x \notin G\left(y^{*}\right)$ for all $x \in E \backslash K$, so $G\left(y^{*}\right) \subset K$, i.e. $\overline{G\left(y^{*}\right)}$ is a compact subset of $X$. On the other hand, because $f(x)-\phi(x, y)$ is lower semi-continuous related to $x, G(y)$ is a closed subset of $X$ for $y \in E$. Theorem 2.3 implies $\bigcap_{y \in E} G(y) \neq \phi$ and since $\bigcap_{y \in E} G(y) \subset G\left(y^{*}\right) \subset K$, so VVI (1.1) has a solution in $E \cap K$.

The following theorem has been proved by S. Park [16] for scalar functions in compact $G$ convex spaces. In addition to extending to vector mode, we have eliminated the condition of compactness from space.

Theorem 3.3. Let $(X ; \Gamma)$ be a $G$-convex space and $\phi, \psi: E \times E \rightarrow V$ and $f: E \rightarrow V$ are functions satisfying

1. $\phi(x, y) \in \psi(x, y)+P$ for each $(x, y) \in E \times E$ and $\psi(x, x) \in P$ for all $x \in E$.

2. For each $x \in E, \psi(x, y)+f(y)$ is cone-quasi-convex related to $y$.

3. For each $y \in E, f(x)-\phi(x, y)$ is lower semi-continuous related to $x$.

4. There exists a nonempty compact subset $K$ of $X$ and a point $y^{*} \in X$ such that

$$
\phi\left(x, y^{*}\right)+f\left(y^{*}\right)-f(x) \in-\text { int } P \quad \text { for all } x \in E \backslash K \text {. }
$$

Then there exists an $\bar{x} \in X \cap K$ such that

$$
\phi(\bar{x}, y)+f(y)-f(\bar{x}) \in P \quad \text { for all } y \in E
$$

Proof. We define the set-valued maps T, $G: E \rightarrow 2^{E}$ as follows

$$
\begin{aligned}
& G(y)=\{x \in E: \phi(x, y)+f(y)-f(x) \in P\} \\
& T(y)=\{x \in E: \psi(x, y)+f(y)-f(x) \in P\} .
\end{aligned}
$$

According to assumption (1), we have $T(y) \subset G(y)$ for all $y \in E$.

By a similar argument as in the proof of Theorem 3.2, $T$ is a generalized KKM mapping. So, $G$ is a generalized KKM map. It follows from condition (3) that $G$ is closed valued map. Condition (4) implies that $x \notin G\left(y^{*}\right)$ for all $x \in E \backslash K$, therefore $G\left(y^{*}\right) \subset K$, then $G\left(y^{*}\right)$ is a compact subset of $X$. By Theorem 2.3 we have $\bigcap_{y \in E} G(y) \neq \emptyset$. Moreover, $\bigcap_{y \in E} G(y) \subset G\left(y^{*}\right) \subset K$. So, the solution of VVI (1.1) is in $E \cap K$. 
Now, we present a Minty type theorem in $G$-convex spaces. The so called Minty vector variational inequality (in short MVVI) is to find $\bar{x} \in E$ such that

$$
f(y)-\phi(y, \bar{x})-f(\bar{x}) \in P \quad \text { for all } y \in E .
$$

Definition 5. The function $\phi: E \times E \rightarrow V$ is said to be cone-monotone if $\phi(x, y)+\phi(y, x) \in$ $-P$ for any $x, y$ in $E$.

In addition, if $\phi$ also satisfies the condition $\phi(x, y)+\phi(y, x)=0$ if and only if $x=y$, then $\phi$ is said to be strictly cone-monotone.

We explain the relationships between the solution set of VVI and MVVI.

Theorem 3.4. Suppose that $\phi: E \times E \rightarrow V$ is a monotone function with $\phi(x, x) \in P$ for all $x \in E$ and $f: E \rightarrow V$ be a function that the following conditions are fulfilled:

1. for each $y \in E, f(\cdot)-\phi(\cdot, y)$ is lower semi-continuous on $\Gamma-\operatorname{Co}\left\{x_{1}, x_{2}\right\}$ for each $x_{1}, x_{2} \in$ E

2. for each $x \in E, f(y)+\phi(x, y)$ is cone-w-convex in $y$.

Also, we suppose that $\Gamma-\operatorname{Co}\left\{x_{1}, x_{2}\right\}$ for any $x_{1}, x_{2} \in E$.

Then $\bar{x} \in E$ satisfies

$$
f(y)+\phi(\bar{x}, y)-f(\bar{x}) \in P \quad \text { for all } y \in E
$$

iff

$$
f(y)-\phi(y, \bar{x})-f(\bar{x}) \in P \quad \text { for all } y \in E
$$

Proof. For each $y \in E$, let

$$
\begin{aligned}
& G(y)=\{x \in E: f(y)+\phi(x, y)-f(x) \in P\} \\
& H(y)=\{x \in E: f(y)-\phi(y, x)-f(x) \in P\} .
\end{aligned}
$$

It is sufficient to prove that $\bigcap_{y \in E} G(y)=\bigcap_{y \in E} H(y)$. First, suppose that $\bigcap_{y \in E} G(y) \neq \emptyset$. For $\bar{x} \in \bigcap_{y \in E} G(y)$, we have

$$
f(y)+\phi(\bar{x}, y) \in f(\bar{x})+P \quad \text { for all } y \in E
$$

Since $\phi$ is monotone then we can deduce $-\phi(y, \bar{x}) \in \phi(\bar{x}, y)+P$ for all $y \in E$. So

$$
f(y)-\phi(y, \bar{x}) \in f(y)+\phi(\bar{x}, y)+P \quad \text { for all } y \in E
$$


By (3.1), (3.2) we obtain

$$
f(y)-\phi(y, \bar{x}) \in f(\bar{x})+P \quad \text { for all } y \in E .
$$

Therefore $\bigcap_{y \in E} G(y) \subset \bigcap_{y \in E} H(y)$. We claim that $\bigcap_{y \in E} H(y) \subset \bigcap_{y \in E} G(y)$. Suppose there exists $\bar{x} \in \bigcap_{y \in E} H(y)$ such that $\bar{x} \notin \bigcap_{y \in E} G(y)$, i.e.,

$$
f(y)-\phi(y, \bar{x}) \in f(\bar{x})+P \quad \text { for all } y \in E
$$

and there exists $\bar{y} \in E$ such that $\bar{x} \notin G(\bar{y})$, or

$$
f(\bar{y})+\phi(\bar{x}, \bar{y}) \notin f(\bar{x})+P .
$$

Let $U=\{z \in \Gamma-\operatorname{Co}\{\bar{x}, \bar{y}\}: f(\bar{y})+\phi(z, \bar{y}) \notin f(z)+P\}$. It is clear that $\bar{x} \in U$ and $\bar{y} \notin U$ (Note that $\phi(\bar{y}, \bar{y}) \in P$ ). Since $f(\cdot)-\phi(\cdot, y)$ is lower semi-continuous on $\Gamma-\operatorname{Co}\{\bar{x}, \bar{y}\}$, then $U$ is open in $\Gamma-\operatorname{Co}\{\bar{x}, \bar{y}\}$.

If $(\Gamma-\operatorname{Co}\{\bar{x}, \bar{y}) \backslash\{\bar{x}, \bar{y}\}) \cap U=\emptyset$, then $U=\{\bar{x}\}$ and so is closed in $\Gamma-\operatorname{Co}\{\bar{x}, \bar{y}\}$. It contradicts the connectedness of $\Gamma-\operatorname{Co}\{\bar{x}, \bar{y}\}$. Therefore, there exists $z^{*} \in(\Gamma-\operatorname{Co}\{\bar{x}, \bar{y}\} \backslash\{\bar{x}, \bar{y}\}) \cap$ $U$, i.e., $z^{*} \in \Gamma-\operatorname{Co}\{\bar{x}, \bar{y}\} \backslash\{\bar{x}, \bar{y}\}$ and

$$
f(\bar{y})+\phi\left(z^{*}, \bar{y}\right) \notin f\left(z^{*}\right)+P .
$$

Setting $y=z^{*}$ in (3.3), we have

$$
f\left(z^{*}\right)-\phi\left(z^{*}, \bar{x}\right) \in f(\bar{x})+P
$$

i.e.

$$
f(\bar{x})+\phi\left(z^{*}, \bar{x}\right) \in f\left(z^{*}\right)-P .
$$

Since $P$ is pointed, so (int $P) \cap(-P)=\emptyset$, then

$$
f(\bar{x})+\phi\left(z^{*}, \bar{x}\right) \notin f\left(z^{*}\right)+\operatorname{int} P .
$$

According to condition (2) together with (3.5) and (3.6), we have

$$
f(x)+\phi\left(z^{*}, x\right) \notin f\left(z^{*}\right)+P \quad \text { for all } x \in \Gamma-\operatorname{Co}\{\bar{x}, \bar{y}\} .
$$

Hence, $\phi\left(z^{*}, z^{*}\right) \notin P$, a contradiction. So $\bigcap_{y \in E} H(y) \subset \bigcap_{y \in E} G(y)$.

If $\bigcap_{y \in E} G(y)=\emptyset$, by the above argument it is easy to see that $\bigcap_{y \in E} H(y)=\emptyset$ and so the proof is complete. 
The following is an extension of Theorem (3) in [24]. Note that theorem 3.3 has been proved for scalar valued functions on $H$-spaces. We have generalized it to vector valued functions on $G$-convex spaces.

Theorem 3.5. Let $\phi: E \times E \rightarrow V$ be a monotone function with $\phi(x, x) \in P$ for all $x \in E$ and $f: E \rightarrow V$ be a function. Suppose that the following conditions are fulfilled:

1. there exists a point $y^{*} \in X$ and a compact subset $K$ of $X$ such that

$$
\phi\left(x, y^{*}\right)+f\left(y^{*}\right)-f(x) \in-\operatorname{int} P \quad \text { for all } x \in E \cap K
$$

2. for each $x \in E, f(\cdot)+\phi(x, \cdot)$ is strongly cone-quasi-convex,

3. for each $y \in E, f(\cdot)-\phi(\cdot, y)$ is lower semi-continuous.

Also, we suppose that $\Gamma-\operatorname{Co}\left\{x_{1}, x_{2}\right\}$ for any $x_{1}, x_{2} \in E$.

Then the solutions set of the vector variational inequality (1.1) and MVVI is a nonempty compact subset of $X$. Furthermore, if $\phi$ is strictly cone-monotone, then the solution of VVI (1.1) and MVVI is unique in $E \cap K$.

Proof. Suppose that $S$ is the solution set for VVI (1.1) in $E \cap K$. From Theorem 3.4, we have $\bigcap_{y \in E} G(y)=\bigcap_{y \in E} H(y)$, where $G(y)$ and $H(y)$ are defined by $G(y)=\{x \in E: f(y)+\phi(x, y)-$ $f(x) \in P\}, H(y)=\{x \in E: f(y)-\phi(y, x)-f(x) \in P\}$. By Theorem 3.2, it is clear that $S$ is nonempty and $S=\bigcap_{y \in E} G(y)$. We show that $S$ is compact subset of $X$ in $E \cap K$. According to the assumption (3), $G(y)$ is closed for all $y \in E$, so, $S=\bigcap_{y \in E} G(y)$ is closed. Given the condition (1) and that $P$ is pointed, we have $S=\bigcap_{y \in E} G(y) \subset G\left(y^{*}\right) \subset K$. Therefore, $S$ is nonempty compact subset of $X$ in $E \cap K$.

We now prove that the solution VVI (1.1) is unique in $E \cap K$ if $\phi$ is strictly cone-monotone. Suppose that $x_{1}, x_{2} \in E \cap K$ be two solutions of VVI (1.1) with $x_{1} \neq x_{2}$. Then

$$
f(y)+\phi\left(x_{1}, y\right)-f\left(x_{1}\right) \in P \quad \text { for all } y \in E
$$

and

$$
f(y)+\phi\left(x_{2}, y\right)-f\left(x_{2}\right) \in P \quad \text { for all } y \in E .
$$

Putting $y=x_{2}$ in (3.7) and $y=x_{1}$ in (3.8), respectively, we have:

$$
\begin{aligned}
& f\left(x_{2}\right)+\phi\left(x_{1}, x_{2}\right)-f\left(x_{1}\right) \in P \\
& f\left(x_{1}\right)+\phi\left(x_{2}, x_{1}\right)-f\left(x_{2}\right) \in P
\end{aligned}
$$

By (3.9) and (3.10) we obtain $\phi\left(x_{1}, x_{2}\right)+\phi\left(x_{2}, x_{1}\right) \in P$. Now, by monotonicity of $\phi$, we have $\phi\left(x_{1}, x_{2}\right)+\phi\left(x_{2}, x_{1}\right)=0$, so $x_{1}=x_{2}$. 


\section{Applications}

As applications, we now extend the Browder fixed point theorem, which is presented for setvalued functions in Hausdorff topological vector spaces by Browder [2], to G-convex spaces by using Theorem 3.2 and prove the equivalent relation between the Browder fixed point theorem and vector variational inequality in G-convex spaces without linear structure.

Theorem 4.1. Let $(X ; \Gamma)$ be a $G$-convex space and $E$ be a compact $\Gamma$-convex subset of $X$. Suppose that $T: E \rightarrow 2^{E}$ is a set-valued mapping satisfy

(I) for each $x \in E, T(x)$ is a nonempty $\Gamma$-convex subset of $E$ and for each $y \in E, T^{-1}(y)$ is an open subset of $E$;

Then $T$ has a fixed point in $E$.

Proof. Suppose that the condition (I) is satisfied and $T$ has no fixed point in $E$. So for each $x \in E$, $x \notin T(x)$. For $x, y \in E$ and $a \in V$, with $a \in-$ int $P$, we define

$$
\phi(x, y)= \begin{cases}a, & (x, y) \in \operatorname{graph}(T) \\ 0, & (x, y) \notin \operatorname{graph}(T)\end{cases}
$$

where $\operatorname{graph}(T)=\{(x, y): y \in T(x)\}$. Then for each $x \in E$, we have $\phi(x, x)=0$. Now, we investigate that all conditions of Theorem 3.2 are established. For $\gamma \in V$ and $x \in E$, we have

$$
\{y \in E: \phi(x, y) \in \gamma-\operatorname{int} P\}= \begin{cases}E, & a-\gamma \in-P \\ T(x), & \gamma=0 \\ \emptyset, & a-\gamma \notin-P .\end{cases}
$$

Since $E$ and $T(x)$ are $\Gamma$-convex, then $\phi(x, y)$ is cone-quasi-convex related to $y$. For each $y \in E$ and $\gamma \in V$,

$$
\{x \in E: \phi(x, y) \in \gamma+P\}=\left\{\begin{array}{l}
E, \quad a-\gamma \in P \\
X \backslash T^{-1}(y), \quad \gamma=0 \\
\emptyset, \quad a-\gamma \notin P .
\end{array}\right.
$$

This implies that $\phi(x, y)$ is upper semi-continuous related to the variant $\mathrm{x}$, so $-\phi(x, y)$ is lower semi-continuous related to $\mathrm{x}$. From Theorem 3.2, there exists $\bar{x} \in E$ such that $\phi(\bar{x}, y) \in P$ for all $y \in E$. Since $\phi(x, y) \in-P$ for all $x, y \in E$, we know that $\phi(\bar{x}, y)=0$ for all $y \in E$, i.e., $T(\bar{x})=\emptyset$, which is a contradiction. 
Remark 3. It is easy to see that the condition (I) in the preceding Theorem 4.1 is equivalent to: (II) for each $x \in E, T(x)$ is an open subset of $E$, and for each $y \in E, T^{-1}(y)$ is a nonempty $\Gamma$-convex subset of $E$.

Theorem 4.2. Browder type fixed point theorem (Theorem 4.1) is equivalent to vector variational inequality (1.1).

Proof. We prove Theorem 4.1 by using the special vector variational inequality (1.1). Now we prove the vector variational inequality (1.1) using Theorem 4.1. If the conclusion of Theorem 3.2 is not true, then for each $x \in E$, there exists $y \in E$ such that $f(y)+\phi(x, y) \notin f(x)+P$. Define $T: E \rightarrow 2^{E}$ as follows

$$
T(x)=\{y \in E: f(y)+\phi(x, y) \notin f(x)+P\} .
$$

$T(x)$ is a nonempty $\Gamma$-convex subset of $E$ for any $x \in E$ and for any $y \in E$ we have

$$
T^{-1}(y)=\{x \in E: f(y)+\phi(x, y) \notin f(x)+P\}=\{x \in E: f(x)-\phi(x, y) \notin f(y)-P\}
$$

is an open subset of $E$ (because $f(x)-\phi(x, y)$ is lower semi-continuous related to $\mathrm{x}$, then $\{x \in$ $E: f(x)-\phi(x, y) \in f(y)-P\}$ is closed i.e., $\{x \in E: f(x)-\phi(x, y) \notin f(y)-P\}$ is open). Therefore condition (I) of Theorem 4.1 is established, then there exists $\bar{x} \in E$ such that $\bar{x} \in T(\bar{x})$, i.e., $f(\bar{x})+\phi(\bar{x}, \bar{x}) \notin f(\bar{x})+P$ and so $\phi(\bar{x}, \bar{x}) \notin P$. This is a contraction. Now, the proof is complete.

\section{References}

[1] A. Bensussan, J. L. Lions, Applications des Inèquations variationnelles en Contrôle Stochastique, Dunod, Paris, (1987).

[2] F. Browder, The fixed point theory of multi-valued mappings in topo-logical vector spaces, Math Ann, 177, 183301 (1968).

[3] I. Chen and Sh. Zou and Y. Zhang, New existence theorems for vector equilibrium problems with set-valued mappings, J. Nonlinear Funct. Anal, 2019 (2019), Article ID4.

[4] R Ferrentino, Variational Inequalities and optimization problems. Appl. Math. Scl, 1, 23272343 (2007).

[5] F. Giannessi, theorems of alternative, quadratic programs and complementary problems, Variational Inequalities and complementarity problems and Applications, PP. 151-186. Wiley, chichester (1980). 
[6] F. Giannessi, Vector Variational Inequality and Vector Equilibrium. Kluwer Academic, Dordrecht, (2000).

[7] C. D. Horvath, Some results on multivalued mappings and inequalities without convexity, in: B. L. Lin, S. Simons (Eds), Nonlinear and Convex Analysis, in: Lect. Notes Pure Appl. Math, Marcel Dekker, 1987, pp. 99-106.

[8] C. D. Horvath, Contractibility and generalized convexity, J. Math. Anal. Appl, 156 (1991), 341-357.

[9] B. Knaster and C. Kuratowski and S. Mazurkiewicz, Ein Beweis des Fixpunktsatsez für ndimensionale simplexe, Fund. Math. 14, (1929), 132冈137.

[10] M. Lassonde, "On the use of KKM multifunctions in fixed point theory and related topics", Journal of Mathematical Analysis and Applications, vol. 97, no. 1, 1983, (151-201).

[11] I. I. Mai and Do Van Luu, Optimality conditions for weakly efficient solutions of vector variational inequalities via convexificators, J. Nonlinear Var. Anal, 2 (2018), 347-389.

[12] S. Park and H. Kim, Foundations of the KKM theory on generalized convex spaces, J. Math. Anal. App, 209 (1997), 551-571.

[13] S. Park, Another five episodes related to generalized convex spaces, Nonlinear Funct. Anal. Appl, 3 (1998), 1-12.

[14] S. Park, Fixed Point,Intersection Theorems, Variational Inequlities, and Equilibrium theorems, J. Math. \& Math. Sci. Vol 24, No, 2 (2000).

[15] S. Park and M. P. Chen, Unified approach to variational inequalities on compact convex sets, Nonlinear Anal, 33, (1998), 637-644.

[16] S. Park, Elements of the KKM theory for generalized convex spaces, korean J. comput. \& Appl. Math, 7 (2000), 1-28.

[17] X. Pin Ding, Generalized variational Inequalities and Equilibrium problems in Generalized convex spaces. J. computers and Mathematics with Applications, 38 (1999) 189-197

[18] N. S. Parageorgiou, Nonsmooth analysis on partially ordered vector spaces. 1. Convex case, Pacific J. Math, 107 (1983), 403-458.

[19] G. Stampacchia, Formes bihneares coercitives sur les ensembles convexes. C. R. Acad. Sci. Paris, Ser, 1. Math. 258, (1964). 
[20] K. Tan, K. G-KKM theorem, minimax inequalities and saddle points, Nonlinear Anal, 30 (1997), 4151-4160.

[21] B. S. Thakar and S. Varghesa, Generalized Nonlinear Variational Inequalities. Novi. Sad. J. Math. vol 44, No, 2, 2014, 29-40.

[22] R. U. Verma, Nonlinear Variational and constrained hemivariational inequalities involving relaxed operators, Zeitschrift fur Ang. Math. and Mechanik, 77 (1997), 387-91.

[23] J. Wang, The Existence of Solutions for general variational Inequality and applications in FC-Spaces. Jourral of Inequalities and Applications, (2012), 1-8.

[24] X. Wu and Xian-Zhi Yuan, Nonlinear variational Inequalitoties and Implicit Variational Inequality of Ky Fan Type in H-Spaces, J. Computers and Mathematics with Applications, 38, (1999), 1-8.

Maryam Salehnejad Department of Mathematics, Science and Research Branch, Islamic Azad University, Tehran, Iran.

E-mail: salehnejad_m@yahoo.com

Mahdi Azhini Department of Mathematics, Science and Research Branch, Islamic Azad University, Tehran, Iran.

E-mail: mahdi.azhini@gmail.com 